\title{
PÄDAGOGIK ÜBER DIE GRENZEN. EIN INTERVIEV MIT PROF. DIETRICH BENNER
}

J.B.: Herr Professor Benner, was hat Sie dazu bewogen, Pädagogik als Ihr besonderes Wissensgebiet zu wählen und zu ergründen?

D.B.: Die Frage ist berechtigt, denn von meiner Ausbildung her habe ich zunächst ja vor allem Philosophie studiert. Als ich in Wien 1964/65 promovierte, bot mir mein österreichischer Doktorvater Erich Heintel eine Assistentenstelle an. Aber der österreichische Staat erlaubte damals nicht, dass ein Deutscher in Österreich eine solche Stelle bekommt. Der zweite Weltkrieg lag noch nicht lange zurück, auch der Staatsvertrag nicht, in dem Österreich als ein neutraler Staat seine Souveränität wiedergewann. Rückblickend ist verständlich, dass Österreich damals seine eigene Nationalität stärken wollte. So musste ich nach Deutschland zurückkehren. Dort hatte ich in meinen beiden ersten Semestern an der Universität Bonn Pädagogik studiert und im Institut für Pädagogik dieser Universität fand ich glücklicherweise meine erste Arbeitsstelle, ausgerechnet bei dem österreichischen Professor Josef Derbolav, der in Bonn eine deutsche Professur innehatte.

M.S.: Was gibt es denn in der Pädagogik überhaupt Anziehendes?

D.B.: Als eine für mich biographisch bedeutsame Antwort auf diese Frage kann ich Ihnen aus meinem Leben zwei Erfahrungen nennen, die für mich besonders wichtig waren. Die erste stammt aus der Oberstufe des Gymnasiums, in Polen sagt man Lyzeum. Ich hatte damals einen sehr guten, heute noch von mir verehrten Deutschlehrer: Herrn Dr. Braun. Im Unterricht bei ihm merkte ich zwei Jahre vor dem Abitur, dass ich denken, meine eigenen Gedanken formulieren und aufschreiben konnte. Nun werden Sie 
fragen, was daran pädagogisch gewesen ist. Dieser Lehrer konnte im Unterricht Fragen stellen, z. B. zu einem Gedicht oder einem Drama oder einer Kurzgeschichte, die die Schüler zum Nachdenken anregten. Durch seine Fragen und durch seinen Unterricht merkte ich damals, dass ich mit seinen Fragen etwas anfangen konnte, das mich an der Sache selbst weiterarbeiten ließ.

Eine ähnliche Erfahrung habe ich später bei meinem Doktorvater in Wien gemacht. Mit seiner Hilfe und Unterstützung konnte ich mich forschend mit einer für neuen Sache so beschäftigen, dass ich anschließend über sie auch mit anderen nachdenken und reden konnte.

Sehr viel später habe ich dann bei Platon gelesen, dass das, was mir zweimal im Leben passiert war, gar nichts besonderes darstellt, sondern auf etwas verweist, die einen bildenden Unterricht ausmachen. In Platons Dialog Symposion lobt der junge Athener Alkibiades den Sokrates, weil er in seiner Nähe Dinge denken und Probleme verstehen und entwickeln könne, die er ohne ihn nicht $\mathrm{zu}$ fassen vermochte.

Mein Einstieg ins Pädagogische war auf diese Weise darüber vermittelt, dass ich an zwei Lehrern die Erfahrung gemacht habe, von einem Lehrer etwas so zu lernen, dass man an der Sache selber weiterarbeiten und mit anderen über sie reflektierend reden kann.

M.S.: Ist es nicht so gewesen, dass die Philosophie Ihnen den Weg zum selbständigen Denken gebahnt hat?

D.B.: Die Philosophie hat mir sehr geholfen. Aber als ich anfing, philosophische Texte zu lesen, habe ich diese zunächst gar nicht verstanden. Ich habe mich gefragt, warum es so lange dauert, wenn ein philosophischer Text einen Gedanken entwickelt. Warum gibt der Autor die Antwort nicht sofort, warum fragt er erst in die eine Richtung, dann in die andere Richtung, warum verwirrt er seinen Leser? Da habe ich schon einen Lehrer gebraucht, der sich für diese Sache, für dieses Verwirrspiel, interessiert und mir 
zeigt, dass die Entwicklung des Gedankens genauso wichtig wie der Gedanke selbst ist. - Auch dies war eine eminent pädagogische Erfahrung.

J.B.: Haben sich Ihre theoretischen Erkenntnisse aus der Pädagogik und wenn ja, wie auf die praktische Erziehung Ihrer eigenen Kinder ausgewirkt?

D.B.: Das ist eine Frage, die meine Frau mir auch oft gestellt hat. Ich glaube schon, dass die theoretische Pädagogik einen Einfluss auf mein praktisches Denken und Handeln gehabt hat und umgekehrt. Es war aber nicht so, dass ich aus der Wissenschaft gelernt hätte, wie man richtig erzieht. Ich habe vielmehr von der Philosophie und von pädagogischen Grundlagentexten gelernt, dass es zum Denken- und Urteilen-Lernen gehört, sich durch Erfahrungen und Fragen irritieren zu lassen. So wurde mein eigenes pädagogisches Nachdenken und Handeln besonders dann irritiert, wenn ich unerwartete pädagogische Erfahrungen mit meinen Kindern machte und mich durch diese dazu aufgefordert sah, etwas Neues zu versuchen und auszuprobieren. Vielleicht kann ich dies an einer kleinen Erziehungsgeschichte mit meinem Sohn Josha erläutern.

Als er zwei Jahre alt war, schlief er abends stets von selbst ein, wenn er ins Bett gebracht wurde. Das war für die Eltern sehr angenehm. Aber so gegen Mitternacht oder kurz nach Mitternacht wurde er zuweilen wach und wollte dann nicht alleine sein. Da kam er zu mir ins Arbeitszimmer und sagte: Papa, ich kann nicht schlafen. Was macht man mit so einem Kind? Ich war irritiert und habe geantwortet: „Schau, ich kann auch nicht schlafen”. Ich habe ihm dann in meinem Arbeitszimmer ein Bücherfach ausgeräumt und es so eingerichtet, dass er in ihm seine eigenen Kinderbücher und Spielsachen aufbewahren konnte. Und wenn er wieder einmal gegen Mitternacht in mein Arbeitszimmer kam, habe ich dann zu ihm gesagt: „Geh' du an deine Sachen, ich gehe wieder an meine 
Arbeit". Und dann haben wir manchmal ein oder zwei Stunden gespielt und gearbeitet. Wenn wir müde wurden, gingen wir schlafen: er in sein Bett, ich zu meiner Frau.

Wenn ich heute manchmal Bücher aufschlage, die damals in der Nähe des Arbeitsfachs meines Sohnes im Bücherregal standen, finde ich in diesen hin und wieder „Eintragungen“, die von meinem Sohn stammen. Er hat offenbar seine Malstifte zuweilen so benutzt, dass er mich, der ich am Schreittisch schrieb und las, nachahmte, und so tat, als könne er lesen und schreiben. Ich habe das damals gar nicht bemerkt. Lesen und Schreiben hat er nicht durch Nachahmung im nächtlichen Arbeitszimmer, sondern in der Schule gelernt. Wir lernen durch Sozialisation nicht das Lesen und Schreiben. Hierfür brauchen wir einen Lehrer, bei dem man die Erfahrung machen kann, dass man in seiner Nähe etwas lernen kann, das man allein oder durch Gewöhnung von anderen nicht lernen könnte.

M.S.: Wie ist es dazu gekommen, dass Sie nun in Warschau lehren?

D.B.: Das ist eine auch für mich nicht restlos geklärte Frage. Ich im Sommer 2008 bekam an der Humboldt-Universität einen unerwarteten Besuch von Frau Prof. Jadwiga Kuczyńska-Kwapisz, der Dekanin der Fakultät für Humanwissenschaften der KardinalStefan-Wyszyński-Universität (UKSW) in Warschau, und von Dr. D. Stępkowski. Herr Stępkowski hatte mich auf diesen Besuch ein bisschen vorbereitet und mir gesagt, vielleicht werde die Dekanin mir einen Lehrauftrag in Warschau anbieten. Aber zu meiner Überraschung stellte die Dekanin mir eine Professur in Warschau in Aussicht und fragte, ob ich für 3 Jahre als Professor nach Warschau kommen würde. Ich habe geantwortet, ich könne ihr die Antwort auf diese Frage am nächsten Vormittag geben, wenn ich über die Möglichkeit, in Warschau zu lehren, mit meiner Frau gesprochen habe. Und am nächsten Morgen habe ich „Ja” 
zu einer möglichen Tätigkeit an der UKSW gesagt. So wurde ich am 1. Oktober 2008 dort ordentlicher Professor und Leiter der Abteilung für die Grundlagen der Allgemeinen Pädagogik. Wie es in Warschau zu der Anfrage an mich gekommen ist, weiß ich bis heute nicht. Darüber müssten Sie die Frau Dekanin fragen.

J.B.: Und nun sind Sie schon über eine gewisse Zeit in Polen tätig. Wie schätzen Sie das Niveau der polnischen Wissenschaft ein? Wie würden Sie sie mit der deutschen vergleichen?

D.B.: Ich würde gern erst ein paar Worte über die Fakultät sagen. In der Fakultät spüre ich eine starke Solidarität unter den Wissenschaftlerinnen und Wissenschaftlern, aber auch zwischen Professoren, Mitarbeitern und nicht-wissenschaftlichen Mitarbeitern. Während der Fakultätssitzungen übersetzt Dr. Stępkowski das Wichtigste für mich, da ich das Polnische nicht verstehe. Ich stelle dann mein Hörgerät ein bisschen lauter und kann seine leise gesprochene Übersetzung verstehen, ohne dass dies andere stört. Auf diese Weise habe ich den Eindruck gewonnen, dass, wenn in der Fakultät geredet wird, die Menschen einander zuhören und dass es keine Intrigen gibt, auch keine Machtkämpfe, sondern - getragen auch durch den Geist der Dekanin - eine große Solidarität in der Sache. Das ist eine enorme Stärke und keineswegs überall selbstverständlich.

Aber es gibt doch auch Schwächen. Es fehlen Arbeitsräume für wissenschaftliches Personal, es gibt keine Ausstattung mit kleinen Etats für die Arbeitsbereiche, es gibt keine Tutoren und keine studentischen Hilfskräfte und es gibt nur ganz geringe Forschungsmittel.

In den drei Jahren, die ich in Warschau tätig bin, habe ich an der Humboldt-Universität bei der Deutschen ForschungsGemeinschaft ungefähr 300.000 Euro für Forschungsprojekte eingeworben, darunter ein Projekt zur Entwicklung eines Instruments zur Beschreibung und Erfassung moralischer Kompetenz bei 
Jugendlichen im Alter von 15 Jahren. Wir haben in Berlin inzwischen ein Kompetenzmodell mit Niveaustufen entwickelt, das jetzt in einer zweijährigen Hauptuntersuchung in Berlin und Brandenburg validiert wird.

$\mathrm{Zu}$ Beginn meiner Warschauer Tätigkeit habe ich gemeinsam mit Dr. Stępkowski einen Schwesterantrag für ein paralleles Projekt beim Polnischen Ministerium für Hochschulbildung und Wissenschaft eingereicht, der den bescheidenen Umfang von ca. 35.000 Euro für zwei Jahre vorsah. Wir wollten eine junge polnische Wissenschaftlerin einstellen und das Instrument nicht einfach auf Polen übertragen, sondern mit dem polnischen Kontext neu abstimmen. Der Antrag wurde in einem Gutachten hochgelobt und dann doch abgelehnt. Er hätte etwa 50\% der in diesem Bereich für ganz Polen zur Verfügung stehenden Finanzmittel verbraucht. Aus polnischer Sicht war es, von der Höhe her, ein unverschämter Antrag. Aber mit weniger Geld - ca. einem Zehntel der in Deutschland bewilligten Summe - kann man solche Projekte auch in Polen nicht bearbeiten.

J.B.: Ich möchte noch eine Nachfrage zu Unterschieden zwischen Polen und Deutschland stellen. In Westeuropa ist es heutzutage sehr populär, dass man vor oder während des Studiums ein Jahr Pause macht und diese Zeit dafür nutzt, die Welt, andere Kulturen kennenzulernen. Bei uns in Polen gibt es diese Möglichkeiten kaum. Wie sehen Sie das? Woher kommt, dass unsere Gleichaltrigen im Ausland durch Reisen die Welt kennenlernen, wir hingegen nach der Mittelschule sofort studieren müssen?

D.B.: Wenn der Wohlstand in Polen einmal deutlich zugenommen hat, wird es auch in Polen vermehrt Jugendliche geben, die zwischen Abitur und Studium reisen, die Welt erkunden, dieses und jenes ausprobieren. Als ich studierte, gab es in Deutschland noch keinen wirklichen Wohlstand. Auch ich habe sofort nach dem Abitur mit 
dem Studium angefangen. Ich glaube aber, dass es in Polen in zehn Jahren auch so sein wird, dass die polnische Jugend mehr reist.

M.S.: Wäre es möglich, das Ausbildungsniveau in Polen und in Deutschland zu vergleichen? Vielleicht wissen Sie, dass sich das polnische Bildungssystem einschließlich des Hochschulsystems seit Jahren in einer Reform befindet. Studienanfänger, die jetzt ihr Studium beginnen, erfahren ganz andere Studienbedingungen, als es noch vor kurzer Zeit der Fall war. Welche Unterschiede und Ähnlichkeiten sehen Sie zwischen Polen und Deutschland in dieser Hinsicht?

D.B.: Es gibt deutliche Unterschiede und es gibt schöne Gemeinsamkeiten. Fangen wir mit den Unterschieden an!

In Polen besteht fast das ganze Studium aus Vorlesungen. In anderen Staaten gibt es neben der Vorlesung Übungen, Seminare, Kolloquien, Tutorien usw. Und es gibt für fortgeschrittene Studenten die Möglichkeit, selber Tutorials und Seminare anzukündigen. Meine Berliner Doktoranden haben alle gelehrt, bevor sie promovierten. In Polen gibt es hingegen fast nur Vorlesungen. In der Vorlesung aber lernen Studierende nicht, das eigene selbständige Denken und Argumentieren auf die Probe zu stellen. Das ist vielleicht der größte Unterschied. Aber es gibt auch sehr erfreuliche Gemeinsamkeiten.

So haben Dr. Stępkowski und ich im letzten Jahr in einer kleinen Gruppe Kandidatinnen, die nach den neuen Studienordnungen an einer BA-Arbeit schreiben, betreut. Es handelte sich um eine Gruppe von nur vier Studentinnen. Wir haben mit diesen individuelle Forschungskonzepte für ein Praktikum sowie eine theoretische Fragestellung für eine empirische Untersuchung ausgearbeitet. Ich will zwei Beispiele nennen.

Das eine ist ein echtes Warschauer Projekt. Es gibt in Warschau die Lebensform von sogenannten Galerianki. Eine Studentin hat dieses Thema für ihre BA-Arbeit ausgesucht. Wir haben mit ihr 
drei wissenschaftliche Ansätze für dessen Bearbeitung ausgewählt. Der eine Ansatz sagt: Galerianki ist eine neue Jugendform, ein Moratorium zwischen Kindheit und Erwachsensein. Die jungen Frauen binden sich nicht an einen bestimmten Mann, sie halten sich die Zukunft offen, machen dabei aber merkwürdige Erfahrungen in diesem Bereich. Die zweite Theorie besagt: Galerianki kann der Beginn einer Karriere sein, die vorübergehend oder auch für längere Zeit in der Prostitution endet. Und die dritte Hypothese lautet, Galerianki ist ein postmodernes Phänomen. Es gibt sie vielleicht in der Wirklichkeit nur selten oder gar nicht. Die Lebensform der Galerianki findet als Simulation im Internet statt.

Wie Sie sehen, haben wir zu Beginn der Arbeit drei sehr verschiedene Hypothesen entwickelt und diese mit pädagogischen und sozialwissenschaftlichen Theorien von Rousseau bis Baudrillard begründet. Die Studentin arbeitet noch an diesem Thema und ist noch nicht fertig. Aus dem polnischen Thema Galerianki wurde auf diese Weise ein interessantes theoretisches und empirisches Forschungsfeld mit systematischen Fragen.

Und natürlich muss man fragen, warum es Galerianki-Phänomene in Warschau, aber nicht in Bukarest, Budapest, Prag, Wien und Berlin gibt. Ich glaube, dass alle drei Hypothesen in gewisser Weise zutreffen. Es wird Beispiele für die Hypothesen eins und zwei geben, aber ich vermute der überwiegende Teil der Phänomene lässt sich dem dritten Typus postmoderner Simulation zuordnen.

Das zweite Thema hängt eng mit dem Berliner DFG-Projekt zusammen, in dem ein Modell mit Niveaustufen zur Erfassung ethischer Kompetenzen von 15jährigen entwickelt wird. Dr. Stępkowski und ich haben das Projekt ja nach der Ablehnung unseres Antrags nicht auf Polen ausweiten können. Aber eine unserer vier Studentinnen hat inzwischen eine BA-Arbeit über dieses Thema geschrieben. Und zwar hat sie nicht einfach das Berliner Projekt wiederholt, sondern eine eigene Fragestellung entwickelt. Sie hat die moralische Kompetenz von polnischen 15-jährigen untersucht und dabei eine Population von Jugendlichen, die im 
Heim untergebracht sind, mit einer Population von Jugendlichen aus einem Gymnasium, einem salesianischen Gymnasium, verglichen. Von den Ergebnissen will ich nur eines nennen.

Die Studentin hat herausgefunden, was zu erwarten war, dass nämlich in weiten Bereichen die Jugendlichen, die das salesianische Gymnasium besuchen, höhere Niveaustufen moralischer Kompetenz erreichen. Aber sie hat auch ein überraschendes Resultat zu Tage gebracht, dass es nämlich Bereiche gibt, in denen die Jugendlichen aus Heimen eine höhere moralische Urteils- und Handlungskompetenz besitzen. Diese Bereiche beziehen sich auf Solidaritätsprobleme in Freundschaften, auf die Abgrenzung von Stehlen und Mundraub sowie auf den Umgang mit Dieben unter Freunden. Ich finde, das ist ein interessantes Resultat. Gestern vormittag hat diese Studentin ihre Arbeit in der Fakultät sehr erfolgreich mit der best möglichen Note verteidigt. Es war das erste BA-Examen in Allgemeiner Pädagogik an der UKSW und ich bin dankbar, dass ich daran mitwirken durfte.

J.B.: Sie sind weltweit tätig und finden auch außerhalb von Deutschland Anerkennung. Die beiden Ehrendoktorate, in Dänemark und Finnland, beweisen, dass Ihr Opus an vielen Universitäten im Ausland bekannt und anerkannt ist. Erfüllt Sie das mit Zufriedenheit?

D.B.: Meine Tätigkeit konzentriert sich auf Europa und auf Kontakte nach China, Taiwan, Japan, die USA und Canada. So habe ich eine Honorarprofessur in Shanghai. $\mathrm{Zu}$ den schönsten wissenschaftlichen Erfahrungen gehört, dass ich im Grunde überall dasselbe wie an der Humboldt-Universität in Berlin lehren kann, und so auch in Shanghai und in Warschau lehre, natürlich mit Hilfe von Kollegen, die selber etwas von der Sache verstehen und sehr gut übersetzen. Es gibt offenbar in modernen Gesellschaften Erziehungsfragen und Erziehungsprobleme, die zwar in sehr unterschiedliche kulturelle Kontexte eingebettet sind, aber in Shanghai, 
Warschau, Berlin, Turku, Vasa oder Kopenhagen sehr ähnlich diskutiert werden. Ich nenne nur ein Beispiel.

Ich habe in Deutschland immer wieder über Theorien der Erziehung gelehrt und geforscht. In Deutschland hatten wir nach dem zweiten Weltkrieg zunächst eine Phase autoritativer Erziehung. Es folgte eine Phase, in der die Jugend skeptisch war gegenüber den Erwachsenen. Dann kam die große Emanzipationswelle, die vieles in Frage stellte. Es folgte eine antipädagogische Phase, in der man vom Ende der modernen Erziehung gesprochen hat. Wenn ich über dieses Thema z. B. in China Vorlesungen halte, dann interessiert das die chinesischen Studenten sehr. Sie sind seit zehn, fünfzehn Jahren in einer Emanzipationsbewegung begriffen. Z. B. suchen sie heute nach einer Lebensform, in der sie ihre Lebenspartner selber auswählen. Noch vor 15 Jahren war das anders. Wenn ein Student ein Examen gemacht hatte, fragte er seinen Professor, ob dieser nicht eine Frau für ihn finden könne. Ich habe meinen chinesischen Kollegen gefragt: Kennen Sie denn so viele Frauen, dass Sie wissen, welche zu welchem Ihrer Studenten passt? Nein, nein, sagte er: Er wähle nicht selber, sondern frage eine Kollegin, ob sie eine Studentin kenne, die zu seinem Studenten passt. Wenn es sich z. B. um einen Studenten handelt, der ein bisschen schwer beweglich, ansonsten aber ehrlich, gründlich und solide sei, dann sage er zu seiner Kollegin: Kennst du vielleicht eine Studentin, die ein bisschen fürsorglich ist, aber auch die Initiative ergreifen kann? Dieser Professor ist mein Freund in Shanghai, er ist berühmt für die Ehen, die er vermittelt hat, weil sie in der Regel lange dauern und weil es nur wenige Scheidungen gibt. In den letzten zehn Jahren kommen zu ihm trotzdem immer weniger und heute überhaupt keine Absolventen mehr, die eine Frau suchen. Die chinesische Jugend ist in einer Emanzipationsbewegung begriffen, was dazu führt, dass ich in Shanghai sehr gut emanzipatorische Themen behandeln kann.

Meine eigene Auffassung ist die folgende: Emanzipation ist kein Bildungsziel. Aber junge Leute müssen sich von ihren Lehrern emanzipieren. Aber diese Emanzipation sollte nicht das 
Resultat der Erziehung, sondern ein Resultat eigener Originalität und Initiative sein. Man kann nicht zur Emanzipation erzogen werden. Man kann nur sich selbst emanzipieren. So lautete auch meine Kritik an der deutschen Emanzipationspädagogik der 60er und 70er Jahre des letzten Jahrhunderts.

Ich habe auch in Warschau versucht, dieses Thema zu behandeln. Aber die Bedeutung war hier eine ganz andere. Dr. Stępkowski und ich haben an der UKSW fast ausschließlich weibliche Studierende. Auf die Frage, ob Frauen sich emanzipieren sollten, hat eine Studentin eine ganz verblüffende Antwort gegeben, die ich nirgends sonst gehört habe. Die Antwort lautete: Darüber, ob eine Frau sich emanzipiert oder nicht, muss der Mann entscheiden. Ich habe erst gar nicht verstanden, was sie meinte; erst durch Nachfragen verstand ich sie. Sie vertrat eine Auffassung, die sich nahe an meiner Kritik an der deutschen Emanzipationspädagogik bewegt. Sie meinte, weder Emanzipation noch Nicht-Emanzipation sei ihr Lebensziel, sie könne sowohl emanzipierte als auch nichtemanzipierte Verhaltensweisen entwickeln. Und wo man sich emanzipiert und wo man sich nicht emanzipiert, das müsse man mit seinem Partner aushandeln. Ist das nicht eine kluge und schöne Antwort?

M.S.: Sie kommen seit drei Jahren regelmäßig nach Warschau. Bestimmt kennen Sie schon unsere Hauptstadt in- und auswendig. Vielleicht haben Sie auch andere Städte Polens besichtigt. Welches sind Ihre Eindrücke? Was denken Sie über die Polen?

D.B.: Vor drei Jahren kannte ich Polen noch gar nicht. Heute fühle ich mich in den Bereichen, wo ich persönlichen Umgang habe, schon etwas zu Hause. Meine Kreise sind einmal die Arbeit mit den Studierenden, dann die Kommunikation in der Fakultät, drittens das Kulturleben in Warschau. Dariusz Stępkowski hat mich zu vielen kulturellen Veranstaltungen mitgenommen: von polnischer Folklore bis hin zu Internationalen Chopin-Festspielen. Der vierte 
Bereich geht darauf zurück, dass ich in Warschau im Kloster lebe. Ich bin bei den Salesianern sehr freundlich aufgenommen worden und fühle mich bei ihnen und ihren Lebensformen fast schon zu Hause. Aber wenn man jetzt meinte, dass ich Polen kenne, so muss ich sagen, dass das nicht der Fall ist. Polen ist für mich voller Geheimnisse. Wenn Sie erlauben, möchte ich einige Beispiele kurz ansprechen.

Das erste Geheimnis ist, dass ich mich frage, wie Polen gegenüber einem deutschen Professor in meinem Alter so freundlich sein können nach den Gräuel, Untaten und Verbrechen, die Deutsche in Polen während des Zweiten Weltkriegs begangen haben. Ein zweites Geheimnis stellt für mich die polnische Geschichte dar. Ich frage mich, warum die Deutschen die polnische Geschichte kaum kennen? Ich erst habe in Polen gelernt, dass Polen eine große europäische Nation mit einer langen Geschichte ist und dass ein polnischer König vor Wien Europa gegen die Türken verteidigt hat.

Dann ist da noch ein anderer Punkt des Verstehens und NichtVerstehens. Es hat mich sehr beeindruckt, wie Polen getrauert hat über den Tod des Präsidenten Kaczyński und der anderen polnischen Repräsentanten, darunter der Rektor der UKSW. Ich habe an dieser Trauer auch persönlich teilgenommen. Aber dann habe ich nicht verstanden, wie man während der Trauer so darüber streiten konnte, ob der Präsident auf dem Wawel beerdigt werden darf. Ich habe vor kurzem Krakau besucht und war auf dem Wawel. Der Präsident liegt gar nicht bei den polnischen Königen. Er liegt außerhalb des Bezirks der polnischen Könige, separat an der Seite von Józef Piłsudski, der sich um Polen sehr verdient gemacht hat. Und es wird nicht nur des Präsidenten, sondern mit einer Tafel aller Opfer von Smoleńsk gedacht. Trauer und Streit in Polen bereiteten mir Probleme, die polnische Nation zu verstehen. Wie kann man so trauern und dann so streiten? Vielleicht ist die polnische Beziehung von Trauer und Streit ja so etwas wie eine demokratische Tugend? Ich weiß es nicht. 
Es gibt noch ein weiteres Geheimnis. Wenn man von Deutschland kommt und vielleicht die ostdeutschen Verhältnisse kennengelernt hat, muss man feststellen, dass Polen trotz des Sozialismus ein katholisches Land geblieben ist. Aber auch in Polen findet heute ein Prozess der Säkularisierung statt, in dem man lernt, dass Recht, Religion, Kunst, Wissenschaft und Politik unterschiedlichen Logiken des Handelns und Denkens folgen. Von daher ist Polen für mich ein Ort einer Modernisierung ganz neuer Art, einer reflexiven Modernisierung, die nicht mit dem Verlust der Religion, sondern der Veränderung von Religion einhergeht.

Aus deutscher Sicht frage ich mich neuerdings gelegentlich, was Polen aus der Geschichte der polnischen Teilungen in die europäische Geschichte einbringen kann. Polen ist für mich ein Land, das so oft unter Österreich, Russland und Preußen ,aufgeteilt", oder treffender formuliert, von diesen Mächten annektiert worden ist, dass die Sorge um seine Einheit heute eine nationale und europäische Sorge zugleich sein muss. Aber gibt es in Polen heute die Sorge um eine neue polnische Teilung? Stellen wir uns vor, es würde ein Atomkraftwerk an der Ostsee gebaut und es würde dort geschehen, was in der Ukraine und in Japan schon geschehen ist! Polen würde auf Jahrtausende ein geteiltes Land sein, ein Land ohne bewohnbaren Ostseestrand.

J.B.: Ich möchte Sie nun über Ihr Konzept einer Allgemeinen Pädagogik befragen. Gibt es einen gemeinsamen Nenner zwischen Erfahrung, Wissen und Wissenschaft und Pädagogik?

D.B.: Man könnte einen gemeinsamen Nenner von Erfahrung, Wissen, Wissenschaft und Pädagogik darin sehen, dass in allen vier Bereichen negativen Erfahrungen eine große Bedeutung zukommt. Man erkennt gewöhnlich den positiven Erfahrungen eine größere Bedeutung als den negativen Erfahrungen zu. Aber die negativen Erfahrungen sind genauso wichtig. Bevor das Kind ein erstes Mal das mit Händen greift, was es sieht und greifen möch- 
te, hat es viele negative Erfahrungen bei der Koordination von Tastsinn und Gesichtssinn gemacht und immer wieder daneben gegriffen. In der Fähigkeit, aus negativen Erfahrungen zu lernen, könnte ein gemeinsamer Nenner von Pädagogik, Erfahrung, Wissen und Wissenschaft liegen.

In meiner Allgemeinen Pädagogik habe ich vier Grundbegriffe des Pädagogischen unterschieden, die für die Interpretation negativer und positiver Erfahrungen wichtig sind. Der erste Grundbegriff besagt: jeder Mensch ist bildsam, er kann negative Erfahrungen machen und aus ihnen lernen. Der zweite Begriff besagt: damit das möglich wird, muss man Kinder zur Selbsttätigkeit auffordern. Der dritte Begriff meint: damit die Kinder in unserer Gesellschaft in Auseinandersetzung mit vorgegebenen Aufgaben lernen können, muss man Aufgaben unserer Gesellschaft in solche für Kinder transformieren. Und der vierte Grundbegriff besagt: der Erziehungsprozess ist genauso wichtig wie das Erziehungsresultat. Erziehung ist nicht nur ein Mittel für das Erreichen eines Ziels, sondern Erziehung ist ein Weg, der konstitutiv für das Ziel ist. So ist moralische Erziehung ebenso so wichtig wie die Bildung und Entwicklung von Moral. Man kann nicht sagen, die Moral ist wichtiger als die moralische Erziehung. Auch die umgekehrte Annahme wäre falsch. Darüber hinaus gilt, dass die Moral und die moralische Erziehung nicht wichtiger sind als die Religion, die Wissenschaft, die Politik oder die Ökonomie. Sie alle stehen in keinem hierarchischen Verhältnis sondern lassen sich nicht-hierarchisch ordnen.

Wenn man das weiter denkt, kann man zu einer überraschenden Schlussfolgerung kommen, die die Aufgaben des öffentlichen Bildungssystems betrifft. Verschiedene Staaten haben versucht, die Erziehung in den Dienst einer Staatsideologie zu stellen. Demokratische Staaten dürfen das nicht. Das, worüber in einer modernen Gesellschaft in Politik, Ökonomie, Religion, Kultur und Wissenschaft gestritten wird, darf durch Erziehung nicht entschieden werden. Die Erziehung muss die nachwachsende Generation in diesen 
Streit einführen, aber darf den Streit nicht entscheiden. Sonst wären die Erzieher und Lehrer die eigentlichen Politiker im Staate. Das aber darf in einer Demokratie, in der die politische Willensbildung Aufgabe der Einzelnen und der ganzen Nation ist, nicht der Fall sein.

M.S.: Könnten Sie Stellung nehmen zu der in Polen sehr verbreiteten Idee der sog. stressfreien Erziehung?

D.B.: Ohne Stress zu erziehen ist eine freundliche, alte reformpädagogische Vorstellung. Aber wir unterscheiden ja zwischen positivem und negativem Stress. Der negative Stress macht müde, inaktiv, man kann unter ihm nichts neu aufnehmen. Der positive Stress fordert heraus, konfrontiert mit Neuem. Ich würde sagen, Erziehung muss zum Lernen herausfordern, schwierige Fragen stellen, von den Lernenden Anstrengungen verlangen und sie muss vor allem Fehler, Irrtümer, Probleme und Fragen bearbeiten und den Umgang mit pädagogischen Schwierigkeiten kultivieren und sogar gelegentlichen Nichterfolg zulassen. Durch die negative Erfahrung, dass ich eine Mathematikaufgabe nicht lösen kann, kann das Interesse an Mathematik wachsen. Das bedeutet, dass wir negativen entmutigenden Stress in der Erziehung vermeiden, aber Fehler, Irrtümer, Anstrengungen, Probleme und Fragen zulassen und bearbeiten müssen. Ohne den positiven Stress negativer Erfahrung geht es nicht. Aber ich würde dafür lieber nicht das Wort Stress, sondern den Begriff Anstrengung verwenden.

J.B.:WoherkommenIhrerMeinungnachdieErziehungsprobleme in unserer Gesellschaft? Ist es heutzutage schwieriger zu erziehen als zu anderen Zeiten?

D.B.: Seit den Tagen des Sokrates wird darüber geredet, dass die Jugend immer schlechter wird und die Erziehung immer schwieriger. Wo kommen die Erziehungsschwierigkeiten her? Es sind immer Schwierigkeiten an Bruchstellen zwischen einer päda- 
gogischen und einer außerpädagogischen Strukturlogik. Gäbe es keine Erziehungsschwierigkeiten, gäbe es keine pädagogischen Entwicklungsprobleme und keinen Fortschritt. Die Bruchstellen zwischen pädagogischen und außerpädagogischen Sachverhalten müssen daher von beiden Seiten beachtet und bearbeitet werden. Ich nenne drei Bereiche.

Der eine bezieht sich darauf, dass wir über Kinder Aufsicht und Zwang ausüben und uns gleichzeitig der Frage stellen müssen, wann und in welchem Umfang dies erlaubt ist. Eine Antwort auf diese Frage lautet: man muss Zwang über Kinder überall dort ausüben, wo sie sonst Schaden erleiden oder sogar zugrunde gehen könnten. So muss man z. B. autoritativ dafür sorgen, dass ein Kind nicht im Meer ertrinkt, aus dem Fenster fällt, mit der Schere in die Steckdose greift, sich an kochendem Wasser verbrennt. Wir müssen aber die Erziehungssituationen so einrichten, dass wir nicht dauernd Zwang ausüben. Darum gestalten wir die Umgebung von Kindern so, dass die Gefährdungen begrenzt und überschaubar sind. Ein zweites Feld ist das des Unterrichts. Es muss Formen von Unterricht geben, in denen Kinder fürs Leben lernen, was sie im Leben selbst unmittelbar nicht lernen können. Unterricht lehrt künstlich, nicht durch unmittelbare Erfahrung. Man muss immer wieder künstliches Lernen interessant machen, damit Lesen und Schreiben, Rechnen und Zeichnen, Geschichte und fremde Sprachen, Naturwissenschaft und Technik und vieles andere mehr fragwürdig und verstanden werden. Ein dritter Bereich des pädagogischen Handelns und der pädagogischen Schwierigkeiten ist die Sorge, dass sich für die nachwachsende Generation ausreichende Möglichkeiten für Partizipation eröffnen und dass es angemessene Übergänge aus pädagogischen Situationen in außerpädagogische partizipatorische Situationen gibt. Dies scheinen mir die drei pädagogischen Felder zu sein, in denen moderne Gesellschaften den Umgang mit pädagogischen Schwierigkeiten kultivieren.

M.S.: Was ist Ihrer Meinung nach ist das wichtigste im Leben? 
D.B.: Ich weiß es nicht. Ich weiß nicht, ob es überhaupt etwas gibt, das man das Wichtigstes im Leben nennen könnte. Wir kennen in einzelnen Lebensphasen unterschiedliche Formen von Wichtigkeit. $\mathrm{Zu}$ Beginn des Lebens ist es sicher wichtig, dass ein Kind Eltern und Bezugspersonen hat, die für es sorgen. Dann braucht es gute Lehrer. Dann muss es einen Beruf lernen. Schließlich suchen Menschen nach einem Partner, der zu ihnen passt. Es ist etwas Schönes, den Weg des Lebens mit einer Person zu gehen, mit der man viele Lebensabschnitte teilt. Es gibt dann so etwas wie eine bewusst gemeinsam geteilte Lebensgeschichte. Aber mit Blick auf all diese Wichtigkeiten gibt es nicht nur Erfolge, sondern auch Scheitern, negative Erfahrungen, die bearbeitet werden müssen. Was da das Wichtige ist, weiß ich wirklich nicht.

Aber ich denke fünf, sechs Punkte kann man nennen. $\mathrm{Zu}$ einem guten Leben gehört, dass man politisch interessiert ist und an politischen Diskursen partizipiert. $\mathrm{Zu}$ einem guten Leben gehört ferner, dass man an der Weiterentwicklung von Sitte und Moral und an moralischen Diskursen teilnimmt. Zu einem guten Leben gehört auch, dass man nicht nur erzogen wird, sondern auch andere erzieht und als Miterzieher Verantwortung übernimmt. $\mathrm{Zu}$ einem guten Leben gehört schließlich, dass man nicht von anderen unterhalten wird, sondern durch Arbeit für sich selbst sorgt. Mit Arbeitslosen kann man keine Demokratie machen. Wer nur Bedürfnisse hat, diese aber nicht befriedigen kann, der kann nur Forderungen stellen. Zu Politik, Moral, Erziehung und Arbeit tritt die Kunst als ein weiterer wichtiger Bereich hinzu. Man muss Welt nicht nur handelnd erleben und gestalten, sondern auch ästhetisch wahrnehmen. Und letztlich würde ich sagen, dass man sein Leben immer auch in dem Bewusstsein führen muss, dass man nicht ewig lebt, sondern sterblich ist und sterben wird und dass es Menschen gibt, die schon gestorben sind und die man in das eigene Leben weiter einbeziehen kann.

Von diesen sechs Bereichen, denke ich, ist kein einziger wichtiger als ein anderer. Es macht keinen Sinn zu sagen: man habe 
zwar keine Arbeit, aber führe statt dessen ein religiöses Leben. $\mathrm{Zu}$ voller menschlicher Existenz und Koexistenz gehören alle sechs Bereiche und auch die Abstimmungsprobleme und Konflikte zwischen ihnen, und manchmal auch Streit.

J.B.: Und ganz zum Schluss möchte ich an Sie die persönliche Frage stellen: was ist Ihr höchstes Glück?

D.B.: Mein höchstes Glück? Wenn ich arbeite, ist mein höchstes Glück die Muße. Wenn ich Muße habe, ist mein höchstes Glück die Arbeit. Aber eigentlich ist mein höchstes Glück, dass ich sehr dankbar sein kann, so viele interessanten Orte kennengelernt zu haben, an denen ich Muße und Arbeit miteinander verbinden konnte. Und einer dieser Orte ist Warschau.

M.S./J.B.: Vielen Dank für das Gespräch.

Tłum. z jęz. niem. Dariusz Stępkowski 